\title{
$\mathrm{M}|\mathrm{R}| \mathrm{S}$ Internet Journal Nitride Semiconductor Research
}

\section{Novel approach to simulation of group-III nitrides growth by MOVPE}

\author{
S. Yu. Karpov ${ }^{1}$, V. G. Prokofyev ${ }^{1}$, E. V. Yakovlev ${ }^{1}$, R. A. Talalaev ${ }^{2}$ and Yu. N. Makarov ${ }^{3}$ \\ ${ }^{1}$ Soft-Impact Ltd (St.Petersburg, Russia), \\ ${ }^{2}$ Ioffe Physical-Technical Institute, \\ ${ }^{3}$ Lehrstuhl für Strömungsmechanik, University of Erlangen-Nürnberg,
}

(Received Wednesday, March 24, 1999; accepted Thursday, May 27, 1999)

\begin{abstract}
Recent studies revealed specific features of chemical processes occurring on the surface of growing group-III nitrides - extremely low sticking probability of molecular nitrogen, low sticking coefficient and incomplete decomposition of ammonia frequently used as the nitrogen precursor. These features (kinetic by nature) result in the growth process going on under conditions remarkably deviated from the gas-solid heterogeneous equilibrium. In this paper we propose a novel approach to modeling of group-III nitride growth by MOVPE taking into account these features. In the model the sticking/evaporation coefficients of $\mathrm{N}_{2}$ and $\mathrm{NH}_{3}$ extracted from independent experiments are used allowing adequate description of the kinetic effects. The model is applied to analysis of growth of binary $(\mathrm{GaN})$ and ternary $(\mathrm{InGaN})$ compounds in a horizontal tube reactor. The growth rate and the solid phase composition are predicted theoretically and compared with available experimental data. The modeling results reveal lower ammonia decomposition ratio on the surface of the crystal as compared to thermodynamic expectations. The developed model can be used for optimization of growth process conditions.
\end{abstract}

\section{Introduction}

Group III-nitrides are materials of great importance suitable for fabrication of green, blue and ultraviolet light emitting diodes (LED), laser diodes [1], and various electronic devices [2]. Progress in these fields are closely related to obtaining epitaxial layers and heterostructures of device quality. The growth techniques widely employed for this purposes are Molecular Beam Epitaxy (MBE) and Metal Organic Vapor Phase Epitaxy (MOVPE) [3] (the latter is the basic growth technique for large-scale production of blue LEDs).

Despite considerable empirical knowledge accumulated by many research groups, clear understanding of the relative role of different physical mechanisms governing the growth process is not yet reached. This may be related to complex surface kinetics occurring in the case of group-III nitrides that differentiates these materials from other III-V compounds.

It was found in early studies that thermal decomposition of $\mathrm{GaN}$ is of strong non-equilibrium character [4], [5]. Extremely high thermal stability of group-III nitrides [4] and catalytic effect of the liquid phase on $\mathrm{GaN}$ vaporization rate [5] were evidences that kinetic effects play a significant role in the processes on the surfaces of group-III nitrides.

The most promising results in the field of group-III nitride growth either by MOVPE or by GSMBE were achieved using $\mathrm{NH}_{3}$ as the nitrogen precursors. In spite of the fact that efficiency of $\mathrm{NH}_{3}$ is several orders of magnitude greater than that of $\mathrm{N}_{2}$, it is still low and, therefore, requires using high V/III ratios. The recent studies [6], [7] carried out under MBE conditions show that the ammonia reaction efficiency is about several per cent under typical growth temperatures (in particular, $4 \%$ at $870{ }^{\circ} \mathrm{C}$ [7] ).

Thermodynamic analysis of various nitrogen precursors used for GaN growth by MOVPE was reported in paper [8]. The calculations show that ammonia should almost completely dissociate into inactive $\mathrm{N}_{2}$ and $\mathrm{H}_{2}$ at temperatures as high as $\sim 400^{\circ} \mathrm{C}$. In this case growth of $\mathrm{GaN}$ would not be favored due to the lack of reactive nitrogen precursor. This fact contradicts experimental observations. This means that the pure thermodynamic consideration is not applicable to analysis of the real growth situation. 
Attempts to take into account the non-equilibrium nature of the surface processes were made in a few works [9], [10], [11]. Koukitu and Seki [9] calculated a dependence of the $\mathrm{GaN}$ growth rate on temperature that correlated with the experimental data on MBE growth of $\mathrm{GaN}$. However, their results were obtained by excluding from consideration the dominant pathway of nitrogen desorption from the surface - associative desorption of $\mathrm{N}_{2}$ (actually in ref. [9], it was assumed that only atomic nitrogen can desorb from the surface of $\mathrm{GaN}$ ). Obviously, this assumption disagrees with the numerous data of mass-spectrometric measurements (see, for example, [4]).

A modification of the thermodynamic approach was proposed to study growth of InGaN ternary compounds by MOVPE [10] and GaN by Hydride Vapor Phase Epitaxy (HVPE) [11]. To take into account the non-equilibrium nature of the ammonia decomposition on the growth surface an adjustable parameter, $X$, was introduced as the stoichiometric coefficient into the heterogeneous cracking reaction of $\mathrm{NH}_{3}$ :

$$
\mathrm{NH}_{3}=(1-X) \mathrm{NH}_{3}+X / 2 \mathrm{~N}_{2}+3 X / 2 \mathrm{H}_{2}
$$

Then a fitting procedure was applied to determine the value of $X$. Obviously, such an approach can be valid only for a narrow range of process parameters, because it is not related to any realistic physical base. In addition it does not allow one to get insight into mechanisms underlying the growth process of group-III nitrides.

An approach allowing one to account for the surface kinetics related to group III-nitrides was developed in our previous studies and applied to modeling of $\mathrm{GaN}$ growth by MBE [12] [13]. The approach is based on introducing a temperature dependent sticking/evaporation coefficient of molecular nitrogen on group-III nitride surfaces into the quasi-equilibrium model of the MBE growth process. The values of the sticking coefficients were extracted from independent experimental data on Langmuir evaporation of binary nitrides in vacuum. Such a way allowed us to get adequate understanding of the main growth trends reported in the literature. But generalization of this approach to the case of MOVPE growth appears to be non-trivial procedure.

In this paper we report on the novel quasi-equilibrium model of MOVPE growth of binary and ternary group III-nitrides where the non-equilibrium effects discussed above are taken into account. It should be noted that the proposed approach has a general character which makes the model applicable to description of the growth processes where kinetic limitations are impor- tant at the stage of adsorption/desorption of the gas phase species at the surface of the growing crystal.

\section{Model of group III-nitride growth by MOVPE}

Modeling of an MOVPE process requires description of various physical phenomena such as gas mixture flow, heat transfer and species mass transport from the reactor inlet to the substrate. The mathematical models of these processes have been developed for many years and are widely described in the literature (see, for instance, [14]). In this paper we focus on the interaction of a gasphase species with the growing surface of the crystal. As discussed in 1 this topic is a bottleneck in understanding of specific features of the growth process.

Our approach is based on two main assumptions:

A. The atoms in the adsorption layer are nearly in thermodynamic equilibrium with the crystal. This means that the rate of atomic incorporation from the adsorption layer into the crystal (occurring at the step edges) and the rate of the reverse process - crystal decomposition (with atomic release back into the adsorption layer) are much higher than their net difference equal to the growth rate of the crystal.

B. Kinetic effects occurring at the stage of adsorption/desorption can be accounted for by introducing the sticking/evaporation coefficients of the individual species. Generally, these coefficients are dependent on temperature and partial pressures of the species.

Below the formulation of the model is given separately for binary and ternary compounds.

\subsection{Binary compounds}

Let us consider $N_{c}$ gaseous species, named as $S_{i}$, taking part in the growth process and transportation in the gas phase of $N_{e}$ elemental components. Let $N_{r}$ independent heterogeneous chemical reactions occur on the growth surface (generally, $N_{r}=N_{c}-N_{e}$ ). These reactions can be written in a symbolic form

$$
\sum_{i=1}^{N_{c}+1} v_{j i} S_{i}=0, j=1 \ldots N_{r}
$$

where $v_{j i}$ is the matrix of stoichiometric coefficients (positive for the reagents and negative for the products of reactions). The summation in Equation (1) is extended to $N_{c}+1$ reactants since formation of the solid nitride compound $\left(i=N_{c}+1\right)$ has to be accounted for in the chemical reactions as well.

We assume that the adsorption/desorption process can be described by using the Hertz-Knudsen equations, i.e. that the net adsorption rate of the $i$-th species at the surface is expressed as: 


$$
J_{i}=\alpha_{i} \beta_{i}\left(p_{i}-p_{i}^{0}\right)
$$

Here $\alpha_{i}$ is the sticking/evaporation coefficient accounting for kinetic limitations in the adsorption/desorption process, $\beta_{i}=\left(2 \pi m_{i} k T\right)^{-1 / 2}$ is the HertzKnudsen factor for the $i$-th species ( $m_{i}$ is the mass of the molecule, $k$ is the Boltzmann constant), $p_{i}$ is the partial pressure of the $i$-th species near the growing surface,

$p_{i}^{0}$ is the equilibrium partial pressure of the $i$-th species.

Strictly speaking, use of Equation (2) is well justified for the case of single-component crystals and a single-component gas phase. In this case, $p_{i}^{0}$ is the saturated vapor pressure of the species over its solid phase. Here we apply Equation (2) to the case of a binary compound and multi-component gas phase. In

this case, however, the equilibrium partial pressures $p_{i}^{0}$ do not have such straightforward physical meanings. Actually these pressures correspond to a certain gas phase composition resulting in zero net adsorption rate of every species and, therefore, to the thermodynamic equilibrium between the gas phase and the crystal.

Taking into account assumption A, the following mass action law equations could be used if all the species are considered as the ideal gases

$$
\prod_{i=1}^{N_{c}}\left(p_{i}^{0}\right)^{\varphi_{\mu}}=K_{j}(T) \quad, \quad j=1 \ldots N_{r}
$$

Here $K_{j}$ is the equilibrium constant of the $j$-th heterogeneous reaction which is calculated using standard Gibbs energies $G_{i}^{\varnothing}$ of the species taking part in the reaction -

$$
K_{j}(T)=\exp \left(-\frac{1}{R T} \sum_{i=1}^{N_{c}+1} v_{j i} G_{i}^{\varnothing}\right)
$$

with $R$ being the universal gas constant.

We assume also that growth of nitride compound occurs stoichiometrically. This requires the species fluxes to met certain mass balance equations which have the following general form

$$
\sum_{i=1}^{N_{c}} f_{k i} J_{i}=V_{g} x_{k} / \Omega_{s}, \quad k=1 \ldots N_{i}
$$

Here $f_{k i}$ is the matrix showing how many atoms of the $k$-th elemental component is contained in the molecule of the $i$-th species, $x_{k}$ is the vector showing how many atoms of the $k$-th elemental component is contained in the solid phase, $\Omega_{s}=M_{s} / \rho_{s} N_{A}$ is the molecular volume of the crystal, $M_{S}$ is the molar mass, $\rho_{S}$ is the density of the crystal, $N_{A}$ is the Avogadro number, and $V_{g}$ is the growth rate of the crystal.

In accordance with assumption B the Equation (2)Equation (5) should be added by the relationships between the sticking coefficients $\alpha_{i}$, growth temperature $T$ and the partial pressures $p_{i}$. In principle such relationships could be derived from the detailed kinetic model of the surface processes. However, at the moment there is a lack of experimental information needed for development of such a model. Therefore, in this paper the only temperature dependent sticking coefficients are used either extracted from independent experiments on free evaporation of binary nitrides in vacuum are used [15], or directly measured [7].

Combining Equation (2)- Equation (5) we get the equation array which can be considered as the boundary conditions relating the species fluxes to their concentrations at the growth surface.

\subsection{Ternary compounds}

A ternary compound $\mathrm{A}_{\mathrm{x}}^{3} \mathrm{~B}_{1-\mathrm{x}}^{3} N$ can be regarded as an alloy of two binary compounds $\mathrm{A}^{3} \mathrm{~N}$ and $\mathrm{B}^{3} \mathrm{~N}$ with atomic concentrations $y_{1}=x$ and $y_{2}=1-x$ respectively. Non-ideality of the alloy is taken into account using the regular solution approximation [16]. According to this approach the mixing Gibbs free energy contains an enthalpy term $\Delta H_{\text {mix }}$ while the entropy of mixing $\Delta S_{\text {mix }}$ is the same as in the case of ideal alloy

$$
\begin{gathered}
\Delta H_{x \rightarrow x}=W \cdot x(1-x) \\
\Delta S_{\text {mix }}=R T \cdot[x \ln x+x \ln (1-x)]
\end{gathered}
$$

Here $W$ is the interaction energy of binary constituents in the solid phase.

In the case of the ternary compound one should consider $N_{r}=N_{c}-N_{e}+2$ heterogeneous chemical reactions 


$$
\sum_{i=1}^{N_{c}+2} v_{j i} S_{i}=0, j=1 \ldots N_{r}
$$

with two solid products $\left(i=N_{c}+1, N_{c}+2\right)$ being the binary constituents $\mathrm{A}^{3} \mathrm{~N}$ and $\mathrm{B}^{3} \mathrm{~N}$ respectively. Then Equation (8) should be substituted by the following equation

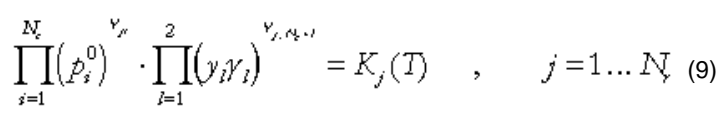

Here

$$
K_{j}(T)=\exp \left(-\frac{1}{R T} \sum_{i=1}^{N_{c}+2} v_{j i} G_{i}^{\varnothing}\right)
$$

and the activity coefficients $\gamma_{1}$ and $\gamma_{2}$ are determined from the equations

$$
\gamma_{1}=\exp \left[\frac{W}{R T} y_{2}^{2}\right], \quad \gamma_{2}=\exp \left[\frac{W}{R T} y_{1}^{2}\right]
$$

As previously, Equation.(9)-Equation (11) should be added by the mass balance equations

$$
\sum_{i=1}^{N_{c}} f_{k} J_{i}=V_{g} \cdot \sum_{i=1}^{2} x_{k}^{l} y_{l} / \Omega_{i} \quad, k=1 \ldots N_{\mathrm{l}}
$$

12)

where $x_{k}^{l}$ are vectors $(l=1,2)$ showing how many atoms of the $k$-th elemental component is contained in the binary compounds $\mathrm{A}^{3} \mathrm{~N}(l=1)$ and $\mathrm{B}^{3} \mathrm{~N}(l=2)$. In addition, one has to use the equation

$$
\sum_{i=1}^{2} y_{l}=1
$$

accounting for normalization of the sum of the concentrations of binary constituents in the ternary compound. And, in accordance to the Vegard law the molecular volume of the solid phase can be calculated as

$$
\Omega_{s}=\sum_{i=1}^{2} \Omega_{l} y_{l}
$$

where $\Omega_{1}$ and $\Omega_{2}$ are the molecular volumes of the binary constituents $-\mathrm{A}^{3} \mathrm{~N}$ and $\mathrm{B}^{3} \mathrm{~N}$ respectively.

\section{Analysis of MOVPE growth of GaN and InGaN}

To demonstrate the workability of the model we apply it to analysis of growth of $\mathrm{GaN}$ and InGaN in a horizontal tube MOVPE reactor. Since the main goal of the work is evaluation of the role of non-equilibrium kinetics in the growth process, a simplified treatment of mass transport in the reactor is used below. In order to relate the partial pressures of species at the inlet of reactor $p^{i n}$ and at the growth surface $p_{i}$ to the flux of the $i$-th species at the surface $J_{i}$, theso-called "diffusive conductivity" $B_{i}$ is introduced by the following way:

$$
J_{i}=B_{i} \cdot\left(p_{i}^{\text {in }}-p_{i}\right)
$$

Obviously, $B_{i}$ as well as $p_{i}$ is dependent on substrate position, reactor geometry, diffusion coefficients of the species, gas flow velocity etc. According to the analytical model of GaAs growth in a horizontal tube reactor, proposed in [17]

$$
B_{i}(g)=2.68 \frac{D_{i}}{b k T} \exp \left(-\frac{2.84 D_{i} q}{b^{2} y_{F}}\right)
$$

where $D_{i}$ is the diffusion coefficient of the $i$-th species, $h$ is the height of the reactor, $z$ is the local coordinate along the susceptor measured from the beginning of the deposition zone, $V_{F}$ is the average gas flow velocity.

Combining Equations (2) and (16) the values of the partial pressures near the growth surface $p_{i}$ as well as the flux of the $i$-th species at the surface $J_{i}$ can be derived as a functions of the inlet pressures $p_{i}^{\text {in }}$ and equilibrium pressures $p_{?}^{0}$ :

(17)

$$
p_{i}=\frac{B_{i} p_{i}^{\text {in }}+\alpha_{i} \beta_{i} p_{i}^{0}}{B_{i}+\alpha_{i} \beta_{i}} \quad, \quad J_{i}=\frac{\alpha_{i} \beta_{i} B_{i}}{\alpha_{i} \beta_{i}+B_{i}}\left(p_{i}^{i n}-p_{i}^{0}\right)
$$

Combining Equation (17) with Equation (2)-Equation (5) in case of binary nitride and with Equation (9)Equation (14) in case of ternary nitride, a closed set of nonlinear equations is obtained in respect to equilibrium 
partial pressures $p_{i}^{0}$, surface partial pressures $p_{i}$ and growth rate $V_{g}$ (as well as to solid phase composition $x$ in case of ternary nitrides). Solution of these equations allows us to calculate the gas phase composition at the surface, growth rate of the crystal (and solid phase composition in the case of ternary nitrides) as a function of the main process parameters: substrate temperature, gas phase composition at the inlet of the reactor etc.

In our analysis we use the well-known Wilke approximation (see, for instance, [14] ) to calculate the diffusion coefficients of gaseous species $D_{i}$. Standard Gibbs energies $G_{i}^{\varnothing}$ of the species necessary to determine the equilibrium constants are taken from [18]. As discussed above, accounting for the adsorption/desorption kinetics of ammonia and nitrogen on/from the surface of growing crystal requires introducing the sticking coefficient of these species into the model. Analysis of interaction of $\mathrm{N}_{2}$ with the surfaces of group III-nitrides was performed in our previous studies [13] [15]. The nitrogen sticking coefficients were extracted there from the independent experiments and evaluated as a function of temperature [15]. The value of the ammonia sticking coefficient $\sim 0.04$ was measured in [7]. The sticking coefficients of all other species are taken to be unity.

First, the proposed model is used to analyze the GaN growth by MOVPE in horizontal tube reactor. The process parameters are taken from Ref. [19]. The following gas phase species are considered: trimethylgallium (TMGa) and ammonia $\left(\mathrm{NH}_{3}\right)$ as the gallium and nitrogen precursors respectively, $\mathrm{H}_{2}$ as the carrier gas, monomethylgallium (MMGa) and $\mathrm{CH}_{4}$ as the products of TMGa gas-phase decomposition, $\mathrm{N}_{2}, \mathrm{Ga}$ and $\mathrm{GaH}$ as the products of surface reactions.

In order to relate the equilibrium pressures $p_{i}^{0}$ to each other the following set of independent heterogeneous reactions is chosen (index $(\mathrm{g})$ indicates the gas phase, index (s) denotes the solid phase):

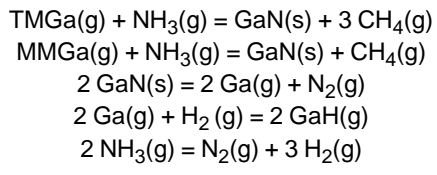

The possibility of adduct formation due to pre-reaction between group-III metalorganic precursors and ammonia is discussed in several papers [19] [20]. This reaction leading to depletion of group-III precursors is generally assumed to be important during MOVPE of $\mathrm{AlN}$ and AlGaN but its relative contribution into the $\mathrm{GaN}$ and InGaN growth process is not clearly under- stood now. Moreover there is lack of reliable data on adduct formation kinetics as well as on the nature of adduct-derived species. That is why we does not include adduct formation into our model. In general this reaction should not be neglected but at present stage of our study we do not take it into account due to the above reasons.

Figure 1 shows the GaN growth rate as a function of growth temperature compared to the experimental data taken from [19]. One can see that in the wide temperature range (up to $\sim 1100^{\circ} \mathrm{C}$ ) growth rate is nearly independent of temperature and growth of $\mathrm{GaN}$ is limited by mass transport of gallium-containing species to the growing surface. At higher temperatures growth rate sharply decreases due to increase of gallium desorption from the surface. One can see that starting from $\sim 700^{\circ} \mathrm{C}$ the calculated and measured values are in a good agreement. The low-temperature decrease of the $\mathrm{GaN}$ growth rate observed in experiment is not reproduced by our model. Under these conditions other mechanisms play important role, and more detailed theoretical consideration of the surface processes is required to describe the growth rate suppression at $T<700^{\circ} \mathrm{C}$.

The dependence of the species partial pressures $p_{i}$ at the surface on the temperature is shown on Figure 2. One can see that at elevated temperatures a remarkable amount of $\mathrm{N}_{2}$ is generated both due to $\mathrm{GaN}$ evaporation and ammonia decomposition at the surface. It should be noted that the equilibrium pressure of nitrogen $p_{N_{2}}^{0}$ is found to be several orders of magnitude greater than the atmospheric pressure. This fact agrees with the experimental observation of an explosion occurred while heating $\mathrm{GaN}$ crystal placed in a sealed quartz tube [21]. Also one can see from Figure 2 that the predicted ammonia partial pressure exceeds its equilibrium value (dashed line) at the temperatures typical for $\mathrm{GaN}$ growth $\left(1000-1100^{\circ} \mathrm{C}\right)$. This difference is the more the less is the total pressure inside the reactor. This result correlates with the observation of non-equilibrium character of ammonia decomposition on the GaN surface discussed in 1 .

Modeling of InGaN growth by MOVPE is based on the experimental data taken from Ref. [22]. The following set of the gas phase species is taken into account: trimethylindium (TMIn), triethylgallium (TEGa) and ammonia $\left(\mathrm{NH}_{3}\right)$ as the indium, gallium and nitrogen precursors respectively, $\mathrm{N}_{2}$ as the carrier gas, In , $\mathrm{Ga}$, $\mathrm{CH}_{4}$ and $\mathrm{C}_{2} \mathrm{H}_{5}$ as the products of group-III precursor pyrolysis, $\mathrm{H}_{2}$, $\mathrm{InH}, \mathrm{GaH}$ as the products of surface reactions. In [22] the horizontal tube AIX-200 reactor with the so called "hot-zone mixing" is used where the gallium and nitrogen precursors are mixed near the sub- 
strate. The gas temperature is there sufficiently high, and adduct formation seems not to occur.

We consider the following independent heterogeneous reactions occurring at the surface while InGaN growing:

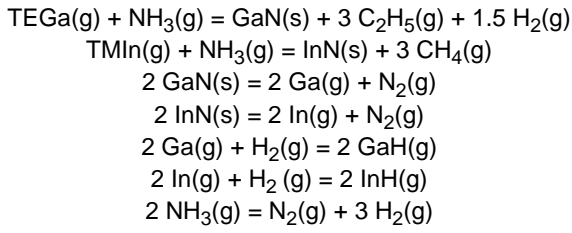

The interaction energy of binary constituents is taken after [23] equal to $25700 \mathrm{~J} /$ mole.

The InN composition in the InGaN ternary compound as a function of substrate temperature under different TMIn/(TMIn+TEGa) input ratios is shown in Figure 3. At low temperatures efficiencies of indium and gallium incorporation into the epitaxial layer are equal, and the InN composition is nearly the same as in the gas phase. But at higher temperatures the indium desorption rate becomes larger than that of gallium and the $\mathrm{InN}$ composition decreases. Such decrease was observed in experiment [22]. Taking into account that no fitting procedure was used in our model, the agreement between experimental data and theoretical predictions looks quite reasonably.

\section{Conclusion}

A novel theoretical approach to modeling of growth of III-group nitrides by MOVPE is proposed accounting for specific kinetics of nitrogen and ammonia interaction with the growth surface. The approach was applied to analysis of growth of $\mathrm{GaN}$ and InGaN compounds in a horizontal tube reactor. Good agreement between theoretically predicted and experimentally measured GaN growth rate and indium composition in InGaN layers is obtained without any fitting procedure.

\section{REFERENCES}

[1] Shuji Nakamura, Gerhard Fasol, The Blue Laser Diode - GaN based Light Emitters and Lasers , (Springer-Verlag, Heidelberg, 1997), .

[2] M. S. Shur, Mater. Res. Soc. Symp. Proc. 483, 15 (1998).

[3] O. Ambacher, J. Phys. D 31, 2653 (1998).

[4] Z. A. Munir, A. W. Searcy, J. Chem. Phys. 42, 4223 (1965).

[5] R. C. Schoonmaker, A. Buhl, J. Lemley, J. Chem. Phys. 69, 3455 (1965).

[6] R. Held, D.E. Crawford, A.M. Johnston, A.M. Dabiran, P.I. Cohen, J. Electron. Mater. 26, 272-280 (1997).

[7] M. Mesrine, N. Grandjean, J. Massies, Appl. Phys. Lett. 72, 350 (1998).

[8] B. Beaumont, P. Gibart, J. P. Faurie, J. Cryst. Growth 156, 140 (1995).
[9] A. Koukitu, H. Seki, Jpn. J. Appl. Phys. 36, L750-L753 (1997).

[10] A. Koukitu, N. Takahashi, T. Taki, H. Seki, J. Cryst. Growth 170, 306-311 (1997).

[11] Heon Lee, Maasaki Yuri, Tetsuro Uedo, James S. Harris Jr, Mater. Res. Soc. Symp. Proc. 423, 233 (1996).

[12] M. V. Averyanova, S. Yu. Karpov, Yu. N. Makarov, I. N. Przhevalskii, M. S. Ramm, R. A. Talalaev, MRS Internet J. Nitride Semicond. Res. 1, 31 (1996).

[13] S. Yu. Karpov, Yu. N. Makarov, M. S. Ramm, MRS Internet J. Nitride Semicond. Res. 2, 45 (1997).

[14] C.R. Kleijn, "Chemical Vapor Deposition Processes", in Computational Modeling in Semiconductor Processing, edited by M. Meyyappan, published by Artech House, Inc.(Norwood), 97(1995)

[15] M.V. Averyanova, I.N. Przhevalsky, S.Yu. Karpov, Yu.N. Makarov, M.S. Ramm, R.A. Talalaev, Mater. Sci. Eng. B 43, 167-171 (1997).

[16] G. B. Stringfellow, Organometallic Vapor-Phase Epitaxy:Theory and Practice, (Academic Press, New York, 1989), .

[17] W. G. J. H. M. van Sark, G. Janssen, M. H. J. M. de Croon, L. J. Giling, Semicond. Sci. Technol. 5, 16 (1990).

[18] I. N. Przhevalskii, S. Yu. Karpov, Yu. N. Makarov, MRS Internet J. Nitride Semicond. Res. 3, 30 (1998).

[19] C. H. Chen, H. Liu, D. Steigerwald, W. Imler, C. P. Kuo, M. G. Craford, M. Ludowise, S. Lester, J. Amano, J. Electron. Mater. 25, 1004 (1996).

[20] A. Thon, T. F. Kuech , Appl. Phys. Lett. 69, 55-57 (1996).

[21] M. R. Lorenz, B. B. Binkowski, J. Electrochem. Soc. 109, 24 (1962).

[22] R. A. Talalaev, E. V. Yakovlev, S. Yu. Karpov, Yu. N. Makarov, O. Schoen, M. Heuken, G. Strauch, Holger Juergensen, MRS Internet J. Nitride Semicond. Res. 4, 5 (1999).

[23] S. Yu. Karpov, Yu. N. Makarov, M. S. Ramm, Sci. Forum 264/268, 1189-1192 (1988).

\section{FIGURES}

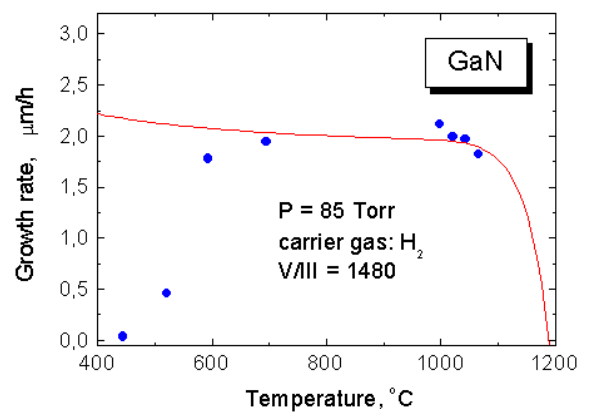

Figure 1. GaN growth rate as a function of substrate temperature. Solid line - modeling results, circles experimental data from [19]. 


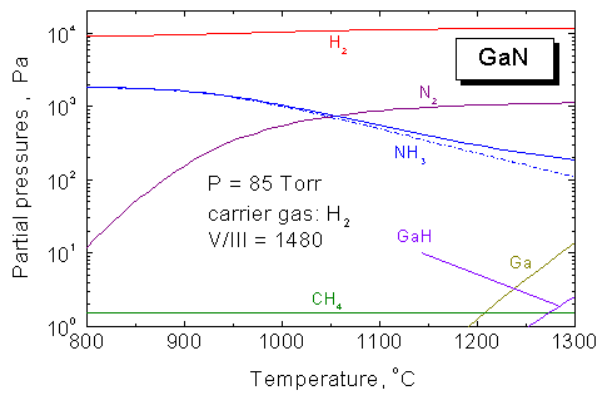

Figure 2. Partial pressures of species near the/ growing surface as a function of substrate temperature. Dashed line thermodynamic pressure of ammonia.

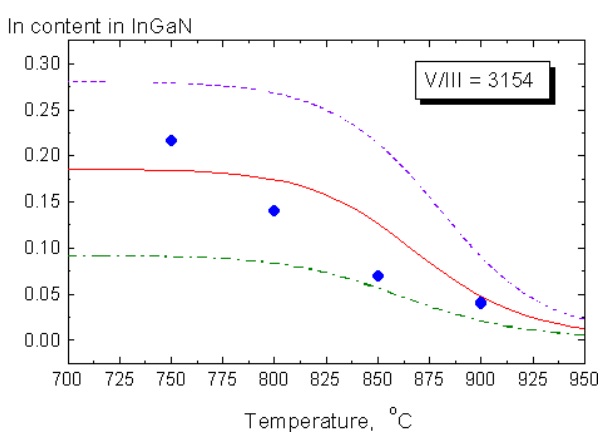

Figure 3. Indium content in InGaN layer as a function of substrate temperature. Violet(dashed) line corresponds to $\mathrm{TMIn} /(\mathrm{TMIn}+\mathrm{TEGa})$ ratio $=0.3$, red line corresponds to TMIn/ $(\mathrm{TMIn}+\mathrm{TEGa})$ ratio $=0.2$, green $($ dashed-dotted $)$ line corresponds to $\mathrm{TMIn} /(\mathrm{TMIn}+\mathrm{TEGa})$ ratio $=0.1$. Points are the experimental data from [22] $(\mathrm{TMIn} /(\mathrm{TMIn}+\mathrm{TEGa})$ ratio $=$ $0.2)$. 